\title{
Meta
}

Journal des traducteurs

Translators' Journal

\section{LARKOSH, CHRISTOPHER (2011) : Re-Engendering Translation. Transcultural Practice, Gender/Sexuality and the Politics of Alterity. Manchester: St. Jerome Publishing, 151 p.}

\section{Georges L. Bastin}

Volume 59, numéro 1, avril 2014

URI : https://id.erudit.org/iderudit/1026484ar

DOI : https://doi.org/10.7202/1026484ar

Aller au sommaire du numéro

Éditeur(s)

Les Presses de l’Université de Montréal

ISSN

0026-0452 (imprimé)

1492-1421 (numérique)

Découvrir la revue

Citer ce compte rendu

Bastin, G. L. (2014). Compte rendu de [LARKOSH, CHRISTOPHER (2011) :

Re-Engendering Translation. Transcultural Practice, Gender/Sexuality and the

Politics of Alterity. Manchester : St. Jerome Publishing, 151 p.] Meta, 59(1),

228-230. https://doi.org/10.7202/1026484ar d'utilisation que vous pouvez consulter en ligne. 
estandarización lingüística, la naturalización, la explicitación y la elevación lingüística. Por último, la parte más innovadora del presente estudio, la comparación de modelos lingüísticos, corrobora aquello a lo que apuntan la revisión histórica y el estudio descriptivo de las traducciones: el texto original en euskera permite otro tipo de pronunciaciones, más modismos y frases hechas y, en definitiva, está más cerca del lenguaje coloquial porque no está sometido a ningún filtro lingüístico; mientras que el texto traducido se distingue por su formalidad, elevación estilística y cohesión. Cierto es que las traducciones se esfuerzan por acercarse al uso coloquial mediante la utilización, muy puntual, de algunos recursos coloquiales de la lengua vasca, pero estas pinceladas no bastan para dotar al modelo lingüístico de las traducciones de la credibilidad que sí se manifiesta en el lenguaje más dinámico y flexible del texto original.

Para concluir, la obra de Barambones se presenta, no solo como una magnífica obra de consulta para aquellos interesados en realizar estudios de TAV basados en corpus con una potente metodología de corte descriptivista, sino también como un trabajo reivindicativo dentro de los estudios de lenguas minoritarias. Es más, se trata de un volumen que invita a la reflexión sobre la incidencia de aspectos socioculturales en el desarrollo de la traducción para el doblaje y, sobre todo, en el desarrollo de los modelos lingüísticos que imperan en esta modalidad de traducción. La obra, además, constituye el número uno de la nueva colección TRAMA, la primera colección europea que versa exclusivamente sobre el campo de la traducción audiovisual, y a la que le damos la bienvenida desde estas líneas, y deseamos, como investigadores de este campo, que nos siga regalando más volúmenes de la calidad y oportunidad del que aquí reseñamos.

Ana Tamayo

Universitat Jaume I, Castellón, España

\section{NOTAS}

1. Nombre de la película (Año de producción)

\section{REFERENCIAS}

Chaves, Ma José (2000): La traducción cinematográfica. El doblaje. Huelva: Servicio de Publicaciones de la Universidad de Huelva.

Martí Ferriol, José Luis (2006): Estudio empírico $y$ descriptivo del método de traducción para doblaje y subtitulación. Tesis doctoral no publicada. Castellón: Universitat Jaume I. Consultado el 4 de abril de 2014, <http://www. tdx.cat/bitstream/handle/10803/10568/marti. pdf? sequence $=1>$.

Zabalbeascoa, Patrick (2008): La credibilidad de los diálogos traducidos para audiovisuals. In: Jenny Brumme, ed. La oralidad fingida: des- cripción y traducción. Teatro, cómic y medios audiovisuales. Madridù: Iberoamericana/ Vervuert, 157-175.

LARkosh, Christopher (2011): Re-Engendering Translation. Transcultural Practice, Gender/ Sexuality and the Politics of Alterity. Manchester: St. Jerome Publishing, 151 p.

On prête rarement attention aux remerciements de l'auteur ou du directeur d'un ouvrage collectif. Ceux-ci sont pourtant éloquents. Ils donnent le ton à un ouvrage revendicateur mais également intimiste. On retrouve ce même ton dans la première des deux exergues, de Holmes (1970) citant Roland Barthes à propos d'un genre d'écriture qui «...ne traite pas du 'monde' mais des formulations linguistiques faites par d'autres; il s'agit d'un commentaire sur un commentaire». Christopher Larkosh fait toutefois remarquer que son intention derrière ces exergues ne signifie ni un assentiment ni un refus de leur contenu, mais bien l'occasion de susciter un débat.

Précisément d'un débat, celui de la conceptualisation de l'intersubjectivité sexuelle, traitent précisément les textes recueillis par Larkosh et réunis dans l'une des dernières parutions de St. Jerome Publishing.

Dans son introduction, et de retour aux exergues, Larkosh met au jour l'appartenance de Holmes à la communauté gay d'Amsterdam, aspect de la vie du célèbre traducteur et traductologue passé sous silence. Et c'est dans le deuxième exergue qu'il trouve le bien-fondé de sa revendication: «There is so much still to be done!» (Holmes 1984). Par exemple, dépasser les délimitations sexuelles courantes, soit les oppositions binaires conventionnelles, pour aborder des positionnements sexuels jugés ambigus tels que bisexuels, hermaphrodites ou transsexuels. En somme, revisiter la traduction sous l'angle du genre, c'est-à-dire, dans ses termes, "re-engendering translation».

Sans se réclamer de l'exhaustivité ni même de la représentativité, Larkosh réunit ici des travaux qui pointent vers de nouvelles possibilités de recherche, vers une véritable "politique de l'altérité» (p. 7) qui consiste à s'identifier à ceux qui sont différents de nous, voire d'eux-mêmes, et à leur donner publiquement un soutien actif et concret (p. 7).

L'ouvrage comprend sept chapitres de traducteurs et chercheurs issus de diverses traditions culturelles, linguistiques et littéraires. Chaque chapitre, en plus de son titre, s'est vu attribuer un thème sauf le dernier. C'est ainsi qu'on trouve les thèmes suivants: "Writing on race and sexuality in the Harlem Renaissance», "Speaking to the 
dead», «Transformations of violence», "Two in translation», "The creation of 'A Lady' » et «Western Others (and 'Other' Westerns)».

Le premier texte se penche sur une série d'écrivaines afro-américaines au cours de la période connue comme la Harlem Renaissance, soit les années 20 et 30 . C'est dans des revues telles que Fire!!, The Crisis, Opportunity, Messenger, Challenge et Black Opals qu'elles font entendre leurs dénonciations de la violence raciale et sexuelle. Des revues émaillées de visuel qui sont autant de sites de résistance et de combat idéologique. Par leur écriture, ces femmes s'élèvent contre les préjugés entourant leur corps et visent à définir leur identité culturelle et sexuelle afin de contrer l'idéologie discriminatoire dominante. Elles publient aussi de nombreux textes méconnus, à compte d'auteur, sans lieu ni date. L'auteure, Annarita Taronna, trace de ces écrivaines un profil bio-bibliographique en insistant sur leurs constantes migrations tant nationales qu'internationales, à Paris principalement, ainsi que sur leur contribution à la tenue de salons littéraires et d'ateliers d'écriture pour jeunes écrivaines. Ces activités de réseautage et d'engagement dans des organisations sociales qui tenaient la question sexuelle comme le facteur le plus fécond pour donner aux femmes un «sentiment d'unité» ont profondément marqué les années 30. Intitulé "Translation as Retelling and Rememory», le texte s'attèle ensuite à la remémoration, rôle assumé par la traduction, notamment celle de certains de ces textes afro-américains traduits en italien par l'auteure. La traduction agit alors en tant que manœuvre rétrospective pour redécouvrir et faire connaître une littérature et une culture féminines, noires et «mineures». Traduire ces textes est une pratique de compréhension interculturelle non neutre qui oppose au «Standard English» prédominant le «black english" périphérique en tant que contre-discours. L'auteure commente alors plusieurs des stratégies utilisées pour rendre en italien un tel sociolecte, notamment la standardisation, le paratexte explicatif et la néologie. L'Italie n'a pas connu d'esclavage ni de discrimination semblables à ceux subis par les écrivaines de la Harlem Renaissance, mais, pour l'auteure, le sort des travailleurs agricoles africains dans les champs de tomates du sud de l'Italie constitue une analogie inspiratrice.

Lisa Bradford, traductrice littéraire argentine et professeure de littérature comparée, traite de la féminisation de la poésie argentine par Juan Gelman comme politique de résistance. Gelman, poète qui débute dans les années 50 et traducteur de Paul Éluard et René Char, convoque dans son œuvre une multiplicité de voix. Il affectionne le collage d'expressions quotidiennes et de l'argot de Buenos Aires avec l'imaginaire du tango. Il pratique la pseudo-traduction et s'adonne à l'imitation tant de compositeurs de tangos que de poètes espagnols tels que saint Jean de la Croix et sainte Thérèse d'Avila. L'exil, qu'il vit à Rome, Paris, Genève et Madrid, lui inspire la nostalgie et l'engagement comme thèmes récurrents. Il adapte aussi les poètes séfardis et se fait, comme le dit Bradford, le «ventriloque» (p. 35) de ces diverses voix. Bradford consacre son chapitre à l'étude du recueil Lettre ouverte (Carta abierta) écrit à la mémoire de son fils et sa belle-fille "disparus» en 1976 durant la dictature de Jorge Videla. À l'instar de Taronna, Bradford traduit son poète, en anglais. Pour ce faire, elle identifie les ressources poétiques de la langue de Gelman : néologismes, féminisation lexicale au moyen de diminutifs, archaïsmes issus de la tradition mystique des poètes espagnols. Elle procède alors à l'auto-analyse de sa traduction qui vise au premier chef à reproduire «la performativité de l'original, la façon dont le texte signifie, afin de rendre la résistance inscrite dans le texte, une résistance non seulement poétique mais politique également.» (p. 37)

Après Harlem et Buenos Aires, Carolyn Shread nous emmène à Haïti. Elle aussi nous relate son expérience de traductrice du roman Les rapaces (1986) de Marie Vieux-Chauvet, un roman allégorique autour de la violence durant la dictature de Duvalier. C'est autant de la violence dans le texte que de la violence dans le processus de traduction que traite Shread. L'auteure s'insurge contre l'idée généralisée selon laquelle, du fait de son impardonnable violence à l'égard d'un «original», la traduction entraîne invariablement une perte. Shread divise son chapitre en deux parties: la première comporte les définitions de la violence et met l'accent sur celle perpétrée à Haïti et l'influence d'un tel contexte sur la traduction. La seconde aborde le rôle du traducteur et l'impact sur la situation cible pour expliquer qu'une traduction métramorphique (voir TTR 20[02]) donne lieu à des choix traductionnels. Le concept de métramorphose, que l'auteure emprunte à Bracha Ettinger (1996), offre «une compréhension nuancée de l'échange au-delà de la sempiternelle appropriation ou identification (intégrer la différence dans le même) et le rejet (exiler la différence)» (p. 63). Par exemple, la violence suscitée par des réactions phalliques à la différence s'en trouvent modulées. Tel est le paradigme qui régit la pratique de traduction de Shread, une alternative aux modèles métaphorique et métonymique.

Le troisième texte, du directeur de l'ouvrage, Christopher Larkosh, porte sur la communication transculturelle multilingue. Concrètement, sur deux auteurs des années 80-90 à Sao Paulo, le premier Néstor Perlongher argentin, l'autre Caio Fernando Abreu brésilien, qui semblent caractériser le «devenir autre» de Deleuze et Guattari 
et dont les thèmes de prédilection sont le multilinguisme, l'homosexualité masculine et le sida. L'article est une pérégrination dans des espaces urbains partagés et d'autres étrangers par ces deux auteurs en quête d'identité. Il n'est toutefois pas nouveau, ce pourquoi nous ne nous y attarderons pas, puisqu'une version en portugais de ce même texte, à quelques expressions près, avait déjà été publiée en 2004 dans la revue Estudios, n 24, de l’Universidad Simón Bolívar à Caracas.

Takayuki Yokota-Murakami, de l'Université d'Osaka, propose un chapitre sur les traductions japonaises de The Lady of the Lake de Walter Scott et de Jane Eyre de Charlotte Brontë à la fin du $19^{\mathrm{e}}$ siècle. Cet essai, illustré par quelques exemples de la traduction et des originaux (en particulier les titres des romans), nous fait découvrir les stratégies d'omission, d'atténuation et d'adaptation des traducteurs qui, souvent par incompréhension, voire inconsciemment, introduisent au Japon une vision occidentale de la relation hétérosexuelle, de l'amour romantique et de l'érotisme. L'auteur met également au jour l'évolution des différents concepts qui définissent la femme japonaise et ceux se rapportant à la beauté physique extérieure et à la beauté spirituelle intérieure. Il rappelle à juste titre que jusqu'en 1890 la distinction entre traduction et adaptation demeurait encore très vague au Japon. Ce serait Futabatei Shimei, en 1965, qui aurait introduit au Japon la notion de fidélité absolue à l'original. Jusque-là, les traducteurs prenaient la liberté de modifier l'original à leur guise ou celle des éditeurs.

Brokeback Mountain en vietnamien est le sujet de l'avant-dernier chapitre par Loc Pham qui souligne d'emblée le caractère postcolonial de son pays et sa résistance perpétuelle à la domination étrangère, donc à l'Autre. Pour lui, dans un tel contexte culturel, l'étranger, ne fût-ce que sa trace, est intolérable, ce qui préfigure l'aspiration obsessive à l'intégrité culturelle pure. Dans la langue, par exemple, qui s'est vue départie du vocabulaire classique chinois (p. 112). Toutefois c'est la résistance à l'Occident capitaliste qui est devenu l'emblème de la construction nationale au Vietnam (p. 113). Les valeurs et les pratiques culturelles contraires à la cohérence et à l'unité culturelles sont critiquées, niées et déportées sur le territoire de l'Autre. Il en est ainsi de l'homosexualité. Quant à la traduction, elle transmet un Autre déficient et devient elle-même l'Autre (p. 116). L'auteur explique alors qu'une traduction sourcière ne sert pas l'homosexualité et par conséquent sa traduction du roman d'Annie Proulx; cette traduction s'en trouverait doublement déplacée: par sa propre étrangeté en tant que thème et par son statut de narration prise en otage par la traduction. C'est pourquoi Loc Pham choisit la traduction cibliste, dont les exemples abondent dans l'histoire de la traduction au Vietnam. La traduction cibliste, n'en déplaise à Berman et à Venuti, n'efface pas les différences culturelles (p. 123) mais représente une stratégie qui permet au silence par rapport à l'identité sexuelle négative d'être entendu. Plutôt que de laisser les lecteurs voyager vers des terres distantes imaginaires, la traduction cibliste de Brokeback Mountain les invite à voyager dans leurs propres sphères culturelles nationales. L'auteur termine en mentionnant rapidement deux aspects de sa démarche: le déplacement temporel de l'original (années 60) vers un Vietnam des années 90 et l'utilisation du dialecte rural du nord du pays. Dommage que l'on n'en apprenne pas plus sur les stratégies utilisées.

L'ouvrage se termine en Inde. Tutun Mukerjee, professeure à l'Université d'Hyderabad, examine deux traductions de Subarnalata de l'écrivaine Ashapurna Debi, l'une en anglais par la traductrice Gopa Majumbar (1997) et l'autre en hindi par le traducteur Hanskumar Tiwari (1978). L'auteure explore la question sexuelle dans l'écriture de l'histoire littéraire et culturelle, et cherche à comprendre la façon dont la traduction s'en charge. La question concrète qu'elle se pose est la suivante: quel genre de sensibilité culturelle est nécessaire pour traduire tous les éléments de l'histoire? Elle répond par le concept d'«excentralité»-dont elle ne donne malheureusement pas de définition - pour les textes «marginalisés et sous-privilégiés». Il ne faut pas nécessairement une traductrice pour traduire un texte féministe, mais plutôt les caractéristiques d'un traducteur sincère et efficace doivent être un engagement profond et rigoureux, de l'empathie, de la sensibilité et de la susceptibilité.

En guise de conclusion, on trouve dans cet ouvrage de nombreuses réflexions sur la «manière de traduire», différente de la manière conventionnelle, par définition occidentale. Nombre de traductologues sont clairement remis en question et plusieurs conceptions de la traduction sont mises à mal. L'ouvrage cherche bel et bien à «ré-engendrer» et à "re-sexiser» la traduction. Des ouvrages d'horizons très divers font l'objet d'analyses fines mais malheureusement de relativement pauvres comparaisons avec les traductions. On comprend bien la nature revendicatrice de l'ouvrage et on appréciera le courage des auteurs. Ils atteignent leur but en faisant prendre conscience au lecteur d'un «monde» trop peu exploré en dépit du potentiel qu'il recèle pour la traductologie. Il faut saluer Christopher Larkosh qui a su réunir de tels essais et poser des questions essentielles.

Georges L. Bastin

Université de Montréal, Montréal, Canado 